\title{
Estimation of genomic breeding values in Gir cattle using Bayesian methods
}

\author{
KR Trivedi, NG Nayee, SG Gajjar, S Saha and RO Gupta
}

Received: 16 April 2021 / Accepted: 18 July 2021 / Published online: 07 September 2021

(C) Indian Dairy Association (India) 2021

\begin{abstract}
The paper evaluates different Bayesian methods in estimating genomic breeding values using a female reference population of Gir breed and assesses their predicting ability and compares the results achieved with those using Genomic Best Linear Unbiased Prediction (GBLUP). In the current study, BayesA, BayesB, BayesC $\pi$, Bayes Lasso, and Bayes-GBLUP methods were used on the same dataset that employed GBLUP and compared the predicting ability of methods using the same five cross-validation data sets. Phenotypic standard 305-day lactation records of 2571 Gir cows extracted from the INAPH database were used in the current study. The milk yield records were pre-corrected for environmental effects. All 2571 cows were genotyped using INDUSCHIP developed by National Dairy Development Board (NDDB). The correlation of SNP effects between BayesC $\pi$ and Bayesian Lasso was 0.99 and their correlations to BayesA and BayesB were 0.814 and 0.685 respectively. The correlation of SNP effects of BayesA and BayesB was 0.977. The correlations between the predicted genomic breeding values and the corrected phenotypic values in all methods were about 0.89 and those between estimated genomic breeding values of different Bayesian methods were equal to one. On comparison of the correlations between estimated genomic breeding values and corrected phenotypic values of five cross-validation data sets for each of five Bayesian
\end{abstract}

National Dairy Development Board, Anand, Gujarat, 388001, India

NG Nayee $(\square)$

National Dairy Development Board, Anand, Gujarat, 388001, India

Email:nileshn@nddb.coop methods, it was observed that the Bayes-GBLUP performed better than GBLUP and also better than all other four Bayesian methods. The other four Bayesian methods BayesA, BayesB, BayesCII, and BayesBL performed slightly better than GBLUP. The correlations among them were more or less the same. From the current study, it could be concluded that Bayesians methods are very useful in estimating both genetic marker effects associated with QTLs and genomic breeding values and that they could be applied in practice for selecting young calves without phenotypic records, based on their genomic breeding values with reasonably high confidence.

Keywords: Bayesian methods, Genomic Breeding Value, Gir cattle, Performance recording

\section{Introduction}

Gir is a very popular and important dairy breed of India. Its native tract is the Saurashtra region in the state of Gujarat housing some 48 percent of the total 2 million female adult Gir cows of the country (Breed Survey, 2013) providing livelihood to a very large number of farmers in the region. Before initiation of a Pedigree Selection (PS) program in 2012 by Sabarmati Ashram Gaushala (SAG) under the National Dairy Plan (NDP) implemented by the National Dairy Development Board (NDDB), little systematic efforts for the genetic improvement of Gir cows had been made. Under the PS project, a very large number of Gir cows had been artificially inseminated (AI) and put under systematic performance recording in the field. With good response from farmers, the PS project was later converted into a progeny testing program during 2017. To leap forward the selection process, NDDB started genotyping recorded Gir cows using the medium density customized microarray chip named as INDUSCHIP developed by NDDB (Saha S. et al., 2020). In the absence of progeny test records of bulls, a female reference population has been built and genomic breeding values (GBV) of both female recorded population and bulls used for AI have been estimated using a Genomic Best Linear Unbiased Prediction (GBLUP) procedure (Nayee N. et al. 2020).

The main objectives of this paper are to evaluate different Bayesian methods in estimating genomic breeding values using 
a female reference population and assess their predicting ability, and to compare the results achieved with those using GBLUP. After the pioneering work by Meuwisen et al. (2001) who developed two Bayesian methods BayesA and BayesB and compared their accuracy of predicting GBV with GBLUP, many alternative methods have been proposed and used in practice for estimating GBV. In both BayesA and BayesB, they assumed the prior distribution of SNP effects normal with a mean zero and a different variance for each SNP, but in BayesB only part of SNPs was considered. BayesC or BayesC $\pi$ is similar to BayesB wherein the prior distribution of SNP effects are assumed normal with a mean zero but unlike BayesB the variance for each SNP is assumed the same(Habier et al. 2011; Verbyla et al., 2009). In Bayes Lasso, the SNP parameters are assumed to have Laplace or double-exponential priors (Park et al., 2008; de los Cambos et al., 2009; Legarra et al.,2011). Different Bayesian methods have been used in many countries for estimating GBVs (Hayes et al., 2009; Colombani et al. 2013, Shopke et al. 2016, S. A. Boison et al. 2017 ). In the current study, BayesA, BayesB, BayesC $\pi$, Bayes Lasso, and Bayes-GBLUP methods were used on the same dataset and compared the predicting ability of methods using the same five validation data sets.

\section{Material and Methods}

The phenotypic and genotypic records, as well as five validation data sets used by Nayee et al. 2020 in their study to estimate genomic breeding values using GBLUP, were used in this study to estimate genomic breeding values using Bayesian methods and compare results among different methods. A brief description of the data sets used is given below.

\section{Phenotypic records}

Phenotypic standard 305-day lactation records of 2571 Gir cows extracted from the Information Network for Animal Productivity and Health (INAPH) database were used in the current study. Monthly test-day records of morning and evening milk yields of individual cows were collected for the entire lactation during the period between 2016 and 2019 by the official milk recorders appointed for the Gir Pedigree Selection program implemented by the Sabarmati Ashram Gaushala under the National Dairy Plan I in the native tract of Gir breed in the Saurashtra region of the state of Gujarat. Animals that had their first record between day 5 and 40 and had subsequent test day records at intervals of 20 to 40 days were kept for the present study. Estimated standard lactation yields falling below $500 \mathrm{Kg}$ or above $6000 \mathrm{Kg}$ were considered as outliers and were dropped from the study.

Not having any de-regressed proofs of bulls or daughter yield deviations (DYD) of bulls, pre-corrected female milk yield records were used as a response variable for estimating SNP effects and genomic breeding values in the present study. The phenotypic records were pre-corrected using the following model: $\mathrm{y}_{\mathrm{ijk} k}=\mathrm{u}+\mathrm{L}_{\mathrm{i}}+\mathrm{S}_{\mathrm{j}}+\mathrm{H}_{\mathrm{k}}+\mathrm{e}_{\mathrm{ijkl}}$

where $y_{\mathrm{ijkl}}$ is the 305 day standard lactation milk yield of $1^{\text {th }}$ animal, $u$ is the overall mean, $L_{i}$ is the fixed effect of $i^{\text {th }}$ lactation, $S_{j}$ is the fixed effect of $j^{\text {th }}$ season and $H_{k}$ is the fixed effect of $k^{t^{j}}$ herd/ village and $\mathrm{e}_{\mathrm{ijk} \mid}$ is the residual effect unaccounted by the model for an individual animal record.

\section{Genotypic records}

All 2571 cows were genotyped using INDUSCHIP having 53K SNPs. Besides, INDUSCHIP genotypes were extracted from 23 bulls that were genotyped using the BovineHD Beadchip (Illumina, San Diego, CA, USA). Using PLINK software (www.cog-genomics.org/plink/1.9/), the SNPs with less than 1\% minor allele frequencies and SNPs having more than $10 \%$ missing genotypes were dropped. Similarly, the animals having less than $90 \%$ SNPs genotyped were also dropped from the analysis. After filtering, the remaining 45875 SNPs were used for the analysis.

\section{Validation data sets}

Five validation data sets were prepared by masking corrected milk yield records of randomly selected $20 \%$ of animals. Thus, in each set different 2057 animals (training set) were used for estimating individual SNP effects, and then using individual SNP effects, genomic breeding values were estimated for the corresponding validation set of 514 animals.

\section{Bayesian methods}

Five Bayesian methods namely BayesA, BayesB, BayesC, Bayesian Lasso, and Bayes-GBLUP were used on the same data set of 2571 animals having corrected milk yield and genotype data, and results were compared. The predicting efficiency of each model was assessed by five-fold cross-validation of $20 \%$ randomly selected animals in each set. The BGLR package of $\mathrm{R}$ was used for estimating different parameters.

In the Bayesian context, the posterior density of a parameter vector is represented as:

$p(\theta / y) \propto p(y / \theta) p(\theta)$

Where $p(y / \theta)$ is the likelihood defined as the density function of data given a set of parameters, and $p(\theta)$ is the prior density of parameters independent of data. The likelihood function for the present study is defined as:

$\mathbf{y}=\mu+\sum_{j}^{j} x j B j+\mathbf{e}$

Where $\mathbf{y}$ is the vector of corrected milk yield; $\mu$ is the overall mean; $J$ is the total number of SNPs; $x j$ the vector of genotypes at SNP $\mathrm{j}$ coded as 0 for AA, 1 for $\mathrm{Aa}$ and 2 for aa; $\mathrm{Bj}$ indicates the 
allelic substitution effect for SNP $\mathrm{j}$ and $\mathbf{e}$ is the vector of residual error. The likelihood is then:

$$
\mathrm{p}(\mathrm{y} / \theta)=\prod_{i=1}^{n} N\left(\mathrm{y}_{\mathrm{i}} / \mu+\sum_{j}^{J} x j B j, \sigma^{2}\right)
$$

The different Bayes methods are defined by their prior distribution $(p(\theta))$ of the SNP effects. The prior distributions assumed for different Bayes methods in the current study are described here. In all models, the residual variance was assigned a scaled inverse Chi-square density.

Bayes A: The prior distributions for marker effects were assumed scale-t density with mean zero and variance $\sigma^{2}{ }_{B j} \sigma^{2}$ Bj were assigned independent and identical density (IID) scaled inverse Chi-square densities with the degree of freedom (DF) assumed to be equal to 5 and the scale parameter assumed to be distributed as gamma density with the rate (r) and shape (s) parameters. $\mathrm{s}$ was assumed to be 1.1 and $r$ was derived assuming $50 \%$ of the total variance attributed to the linear predictors.

BayesB: It is an extension of BayesA with the addition of parameter $\pi$ which is the proportion of non-zero marker effects. The markers were assigned IID priors with mean zero and $\sigma^{2}{ }_{B j}$, and $\sigma^{2}{ }_{B j}$ were assigned similar hyperparameters like in BayesA. $\pi$ was treated as an unknown parameter and assigned Beta prior.

BayesC or BayesC $\pi$ Here the prior distributions for marker effects were assumed normal with mean zero and variance $\sigma^{2}{ }_{B}$. $\sigma^{2}{ }_{B}$ was assigned a scaled inverse Chi-square density with an additional parameter $\pi$. $\pi$ was assigned Beta prior as in BayesB.

Bayes Lasso: Here marker effects were assigned doubleexponential density with independent normal density at first level with mean zero and marker specific variance $\tau^{2}{ }_{j} \times \sigma^{2} . \sigma^{2}$ was assigned scaled-inverse Chi-square density and $\tau^{2}{ }_{\mathrm{j}}$ was assigned IID exponential densities with rate parameter $\lambda^{2} / 2$. At the second level, $\lambda^{2}$ was assigned a gamma distribution with shape $s$ and rate $\mathrm{r}$ parameters. $\mathrm{s}$ was assumed to equal 1.1 and the rate parameter was derived matching the expected portion of variance accounted by the corresponding linear predictor.

Bayesian GBLUP: It is similar to a standard Animal Model GBLUP procedure in which the pedigreed derived numerator relationship matrix $\mathrm{A}\left(\mathrm{a} \sim \mathrm{N}\left(0, A \sigma^{2}\right)\right)$ is replaced with genomic relationship matrix $\mathrm{G}\left(\mathrm{g} \sim \mathrm{N}\left(0, \mathrm{G} \sigma_{\mathrm{g}}^{2}\right)\right.$. Bayesian GBLUP is equivalent of GBLUP where variances $\left(\sigma_{\mathrm{g}}^{2}\right.$ and $\left.\sigma_{\mathrm{e}}^{2}\right)$ are estimated simultaneously along with the estimation of genetic effects $(u)$ unlike in GBLUP where $\mathrm{h}^{2}$ is assumed to be given. An RKHS regression was implemented specifying the Kernel matrix $\mathrm{K}$ as a $\mathrm{G}$ matrix.

Gibbs Sampler was used to estimate the model parameters. Each analysis was based on a chain of 120,000 iterations with an initial 20,000 burn-in cycles and thining equal to 5 .

\section{Comparison of methods}

The comparison of different Bayesian methods was done in two ways one by estimating the measures of fitness of models and the second by measuring the predictability of different methods.

\section{Goodness of fit}

Two goodness of fit measures, one a model complexity measure $\left(\mathrm{P}_{\mathrm{D}}\right)$ reflecting on the effective number of parameters in a model and the other a deviance information criterion (DIC) as proposed by D. J. Spiegelhalter (2002), were used for the comparison of the models. $\mathrm{P}_{\mathrm{D}}$ is defined as:

$$
\mathrm{P}_{\mathrm{D}}=\overline{D(\theta)}-\mathrm{D}(\overline{\theta)}
$$

Where $\overline{D(\theta)}$ is the posterior mean of deviance and $\mathrm{D}(\bar{\theta})$ is the deviance of mean. DIC is defined as:

$$
\mathrm{DIC}=\mathrm{D}(\bar{\theta})+2 \mathrm{P}_{\mathrm{D}}
$$

DIC penalizes for the overfitting of a model by adding $\mathrm{P}_{\mathrm{D}}$ to the deviance statistics and is considered a Bayesian measure of goodness of fit.

The models were also compared by calculating the correlation between the estimated genomic breeding values and the corrected phenotypic values and also by estimating heritability derived from the estimates of residual errors. The proportion of variance explained by the markers is derived by subtracting the error variance from 1 as after standardization of $y$ variable the total variance is 1 .

\section{Predictability of model}

For assessing the predictability of each Bayesian method, five validation data sets were prepared by masking corrected milk yield records of randomly selected $20 \%$ of animals as described earlier. Each set thus had 2057 animals having phenotypic and genotypic records as a training set and the remaining 514 animals having only genotypic records as a validation set. At every run, the training set was used for estimating individual SNPs effects, and the estimated individual SNPs effects were then used for estimating genomic breeding values of the validation set using their genotype data alone. The predictability of each model was assessed by calculating the correlation between the predicted genomic breeding values and corresponding corrected yields of the validation set.

Breeding values of 23 bulls having only genotype data were also estimated separately using just the individual SNP effects estimated under each Bayesian method using the entire female data set of 2571 records. 


\section{Results and Discussion}

Manhattan plots of the estimated posterior SNP mean effects on a $\log$ scale for BayesA, BayesB, BayesC $\pi$, and Bayesian Lasso are given in Figure 1. The Manhattan plots show that the SNP association effects are small and well spread across all chromosomes covering the whole genome. Mean SNP effects of all chromosomes are shown in Figure 2. The SNP mean effects for all chromosomes follow more or less the same pattern in all four Bayesian methods. The correlations of mean SNP effects between different Bayesian methods were more than 0.90 and highly significant. Chromosome No. 29 had the highest and chromosome No.13 had the second-highest SNP mean effects among all Bayesian methods. For illustration, plotting of individual SNP effects of chromosome No. 29 is given in Figure
3. It is seen from the figure that larger SNP effects are similar in all four Bayesian methods. This explains that some clusters of SNPs might be linked with genomic regions influencing milk yield. Density plots of SNP effects are depicted in Figure 4. The density plots of Bayes $\mathrm{C} \pi$ and Bayesian Lasso are almost superimposed on each other and they are different from those of BayesA and BayesB. As expected, the density plots of BayesA and BayesB are very similar in shape. This is further corroborated by comparing the correlations of SNP effects between different methods (Table 1, below the diagonal). The correlation of SNP effects between BayesC $\pi$ and Bayesian Lasso works out to 0.99, whereas their correlations with the SNP effects of BayesA and BayesB are 0.814 and 0.685 respectively. SNP effects of BayesA and BayesB on the other hand are highly correlated with a correlation of 0.977 . All correlations between genomic breeding
Fig. 1 Manhattan plots of SNP effects for different Bayesian methods
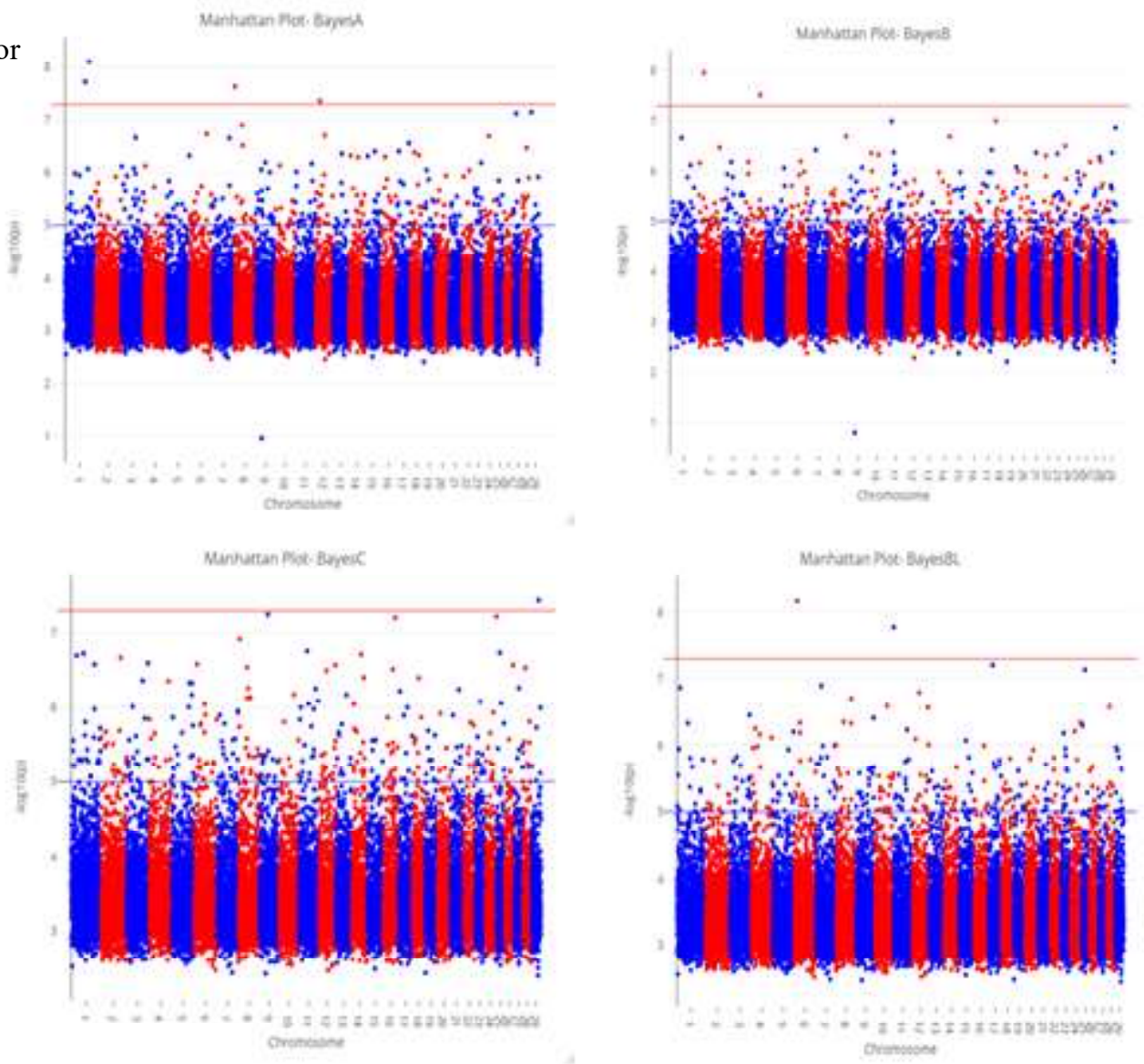

Table 1 Correlations between SNP effects (below diagonal) and estimated genomic breeding values (above diagonal) for different methods

\begin{tabular}{|c|c|c|c|c|c|}
\hline $\begin{array}{l}\text { Bayes } \\
\text { Method }\end{array}$ & BayesA & BayesB & BayesC $\pi$ & BayesBL & GBLUP \\
\hline Bayesian GBLUP 1 & 0.9915 & 0.9911 & 0.9907 & 0.9907 & 0.991 \\
\hline BayesA & 1 & 0.9997 & 0.9990 & 0.9990 & 0.999 \\
\hline BayesB & 0.977 & 1 & 0.9978 & 0.9977 & 0.997 \\
\hline BayesC & 0.814 & 0.685 & 1 & 0.9999 & 0.999 \\
\hline BayesBL & 0.814 & 0.684 & 0.995 & 1 & 0.999 \\
\hline
\end{tabular}


values of different Bayesian methods are also almost equal to one (Table 1, above the diagonal) which indicates that all Bayesian methods are quite consistent. For comparison, the correlations of estimated GBV by GBLUP with those of Bayesian methods are also given in Table 1.

The diagnostic measures applied for comparing the Bayesian methods are given in Table 2. On both the deviance measures $\mathrm{P}_{\mathrm{D}}$ and DIC - the Bayes-GBLUP performed much better than the other Bayesian methods. As in the Bayes-GBLUP, fewer parameters are to be estimated compared to the other Bayesian methods, its $\mathrm{P}_{D}$, which is a measure of model complexity is much lower than those of other Bayesian methods. Among the Bayesian methods other than Bayes-GBLUP, $\mathrm{P}_{\mathrm{D}}$ is the lowest for BayesBL and the highest for Bayes $\pi$. The comparison of DIC among different methods also suggests that the Bayes-GBLUP is superior to other Bayesian methods for this data set. Among the other Bayesian methods, the DICs for BayesC $\pi$ and BayesBL are higher than those for BayesA and BayesB. Considering both $\mathrm{P}_{\mathrm{D}}$ and DIC together, it could be inferred that there is not much difference in model performance among BayesA, BayesB, Bayes $C \pi$, and BayesBL, although BayesBL seems to have a slight edge over others. The comparison on the other two measures - $r$ and $\mathrm{h}^{2}$ - suggests that the performances of all models are

Table 2 Comparison of different Bayesian model parameters

\begin{tabular}{lllll}
\hline Bayesian Model & $\mathrm{P}_{\mathrm{D}}$ & $\mathrm{DIC}$ & $\mathrm{r}$ & $\mathrm{h}^{2}$ \\
\hline Bayes GBLUP & 930 & 6408 & 0.886 & 0.505 \\
BayesA & 949 & 6432 & 0.891 & 0.505 \\
BayesB & 943 & 6431 & 0.889 & 0.504 \\
BayesC $\pi$ & 952 & 6441 & 0.891 & 0.503 \\
BayesBL & 932 & 6452 & 0.888 & 0.497 \\
\hline
\end{tabular}

indistinguishable. $\mathrm{r}$ measures the correlation between the estimated genomic breeding values and the corrected phenotypic values, and $\mathrm{h}^{2}$ is the heritability estimate a ratio of genetic variance, derived from error variance, to the total variance.

The estimated posterior mean of hyperparameter $\pi$ for BayesB and BayesC $\pi$ were $0.439 \pm 0.118$ and $0.415 \pm 0.128$ respectively. The posterior mean of the hyperparameter lambda for Bayesian Lasso was 217. The density plots of $\pi$ of BayesA and BayesB and that of lambada for Bayesian Lasso are given in Figure 3. The scale parameters for scaled-inverse Chi-square distribution were 0.000198 for BayesA and 0.000395 for BayesB and BayesC $\pi$. The degree of freedom taken in each case was 5. The second level rate and shape hyperparameters for BayesA, and BayesB were 506 and $6.71 \mathrm{e}-05$, and 253 and 0.00016 respectively. The shape parameter for BayesBL was 2.82e -06.

The correlations between estimated genomic breeding values and corrected phenotypic values ( $\mathrm{r}$ ) and the regression of corrected milk yield on estimated GBVs (b) (normally referred to as the bias of estimated GBVs) of five cross-validation data sets for all five Bayesian methods are given in Table 3 . The five training and validation data sets used for all five Bayesian methods were the same. For a comparison purpose, the correlations between GBVs and corrected phenotypic values obtained by Nayee et al. 2020 using GBLUP for the same five data set used in the current study are also presented in Table 3. It is seen from Table 3 that the Bayes-GBLUP performed better than GBLUP and also better than all other four Bayesian methods. All four Bayesian methods BayesA, BayesB, BayesC $\pi$, and BayesBL also performed slightly better than GBLUP, however, the correlations for all of them were
Bayes A

Fig. 2 SNP mean effects plots by chromosome number for different Bayesian methods

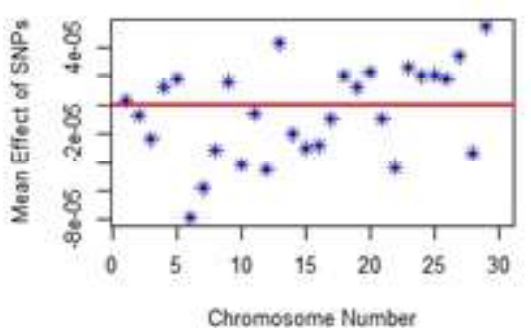

BayesC

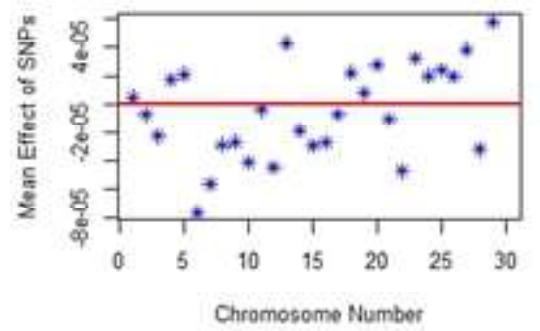

BayesB

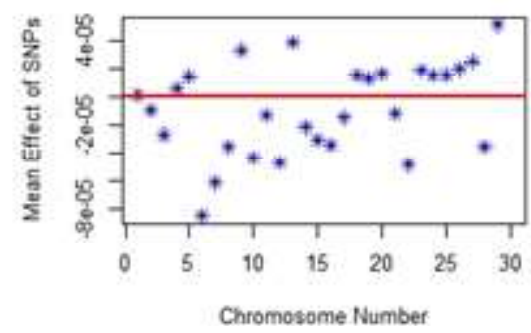

BayesBL

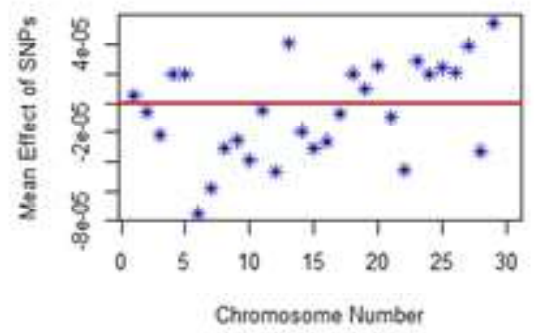


more or less the same. All regression coefficients were around one indicating unbiasedness of estimated GBVs.

The estimated genomic breeding values of 23 bulls used for AI were obtained simply by multiplying their genotype by the estimated SNP effects under each method. The correlations of estimated genomic breeding values of 23 bulls between different methods were almost one.

The main objective of the study was to compare the performance of Bayesian methods with the GBLUP method in estimating genomic breeding values and assess their ability to predict genomic breeding value using the data set of Gir cattle used by Nayee N.et al. 2020. The results of the current study suggest that the Bayesian methods are slightly better than the GBLUP method and could be used in selecting young bull calves and heifers based on their estimated genomic breeding values. Among the Bayesian methods studied, the Bayes-GBLUP performed better than the other Bayesian methods. The performances of the other four Bayesian methods were indistinguishable, although the Bayesian Lasso had a slight edge over the others. Habier et al. (2011) studying different Bayesian methods - BayesA, BayesB (referred to as BayesD $\pi$, BayesB (with known $\pi$, and BayesC $\pi$ in predicting genomic breeding values in North American Holstein observed that accuracies of different methods were similar across all traits and training data sizes and recommended the use of Bayes $\mathrm{C} \pi$ for routine application considering the accuracy and time required in computation. Colombani et al. (2012) while comparing the performance of different Bayesian methods particularly Bayes $\mathrm{C} \pi$ and Bayesian Lasso with pedigree-based BLUP and GBLUP for three traits namely milk yield, fat $\%$ and conception rates in Holstein Friesian and Montbeliarde breed in France concluded that the Bayesian genomic selection methods were slightly more accurate than pedigree-based BLUP, but the gain in the accuracies were limited. The prediction accuracy of BayesC $\pi$ and Bayesian Lasso was slightly better than ssGBLUP but not significantly different. Legarra et al. (2011) in their study
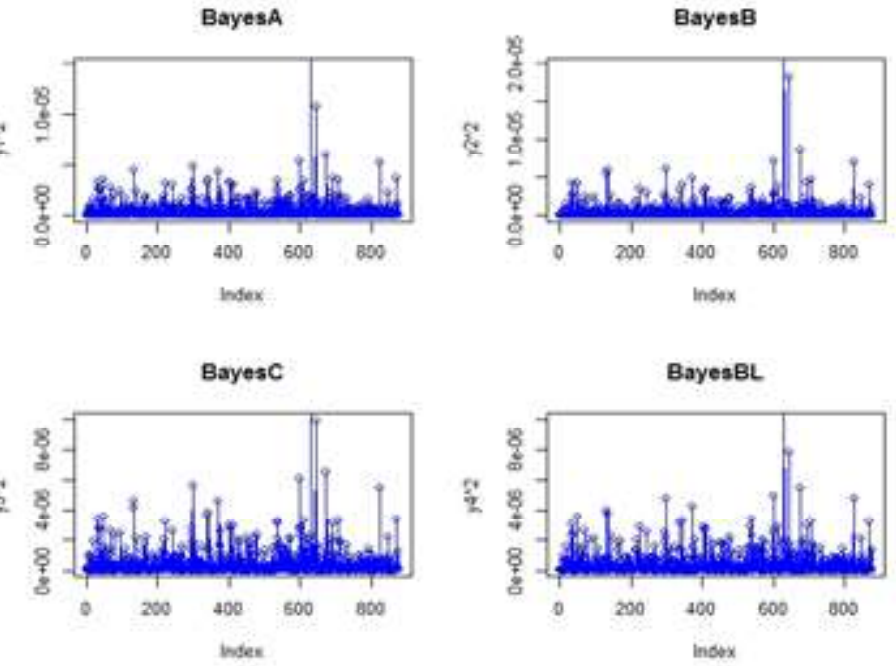

Fig. 3 Plots of individual SNF effects for chromosome No. 29

of Holstein Friesian and Montbeliarde breed in France showed that the Bayesian Lasso performed slightly better than the ssGBLUP and Bayes-GBLUP.

When many QTLs governed a trait, each with a small effect, GBLUP perform better than other Bayesian methods. GBLUP is independent of the number of QTLs associated with a trait. It is observed that as the number of QTLs involved reduces, the Bayesian methods perform better (Daetwyler et al., 2010; Hanni et al., 2012). Milk yield in the Gir population under the current study seems to be controlled by many QTLs as reflected by a large number of SNPs influencing milk yield each having a small effect. As regards BayesGBLUP doing better than GBLUP or ssGBLUP, it is postulated that a simultaneous estimation of genomic breeding values and variance components $\sigma^{2}$ and $\sigma^{2}$ e in BayesGBLUP leads to its better performance than GBLUP wherein the variance components are predetermined (Hanni et al., 2012). When there are many large SNP effects associated

Table 3 Correlations between predicted genomic breeding values and corrected milk yields for different Bayesian methods for randomly drop five record sets

\begin{tabular}{|c|c|c|c|c|c|c|c|}
\hline Records & & GBLUP* & B-GBLUP & BayesA & BayesB & BayesCII & BayesBL \\
\hline \multirow[t]{2}{*}{$\overline{\text { Set } 1}$} & $r$ & 0.433 & 0.444 & 0.445 & 0.447 & 0.445 & 0.444 \\
\hline & $\mathrm{b}$ & & 1.122 & 1.157 & 1.165 & 1.158 & 1.168 \\
\hline \multirow[t]{2}{*}{ Set 2} & $r$ & 0.463 & 0.480 & 0.466 & 0.467 & 0.466 & 0.467 \\
\hline & $\mathrm{b}$ & & 0.955 & 0.954 & 0.957 & 0.956 & 0.970 \\
\hline \multirow[t]{2}{*}{ Set 3} & $\mathrm{r}$ & 0.463 & 0.502 & 0.472 & 0.472 & 0.473 & 0.472 \\
\hline & $\mathrm{b}$ & & 1.126 & 1.060 & 1.063 & 1.057 & 1.065 \\
\hline \multirow[t]{2}{*}{ Set 4} & $\mathrm{r}$ & 0.482 & 0.510 & 0.479 & 0.481 & 0.479 & 0.480 \\
\hline & $\mathrm{b}$ & & 1.101 & 1.052 & 1.054 & 1.059 & 1.071 \\
\hline \multirow[t]{2}{*}{ Set 5} & $r$ & 0.426 & 0.440 & 0.434 & 0.435 & 0.431 & 0.431 \\
\hline & $b$ & & 0.915 & 0.938 & 0.937 & 0.939 & 0.946 \\
\hline
\end{tabular}

*Figures reported by N Nayee et al. (2020); $\mathrm{r}$ is the correlation between estimated genomic breeding values and corrected milk yield, and $\mathrm{b}$ is the regression of corrected milk yield on estimated genomic 
Fig. 4 Density plots of SNP effects, pi, and lambada for different Bayesian methods

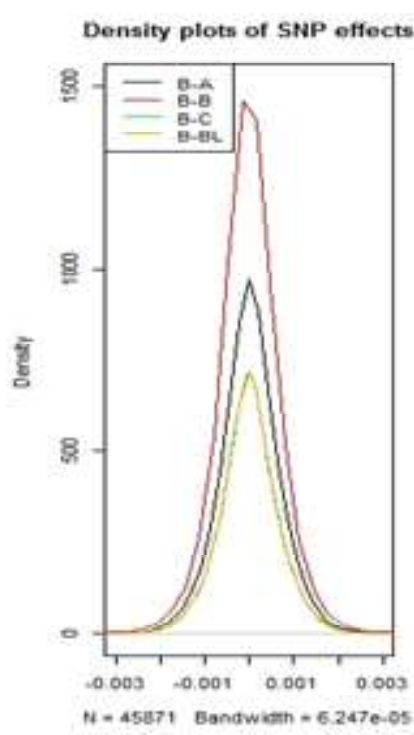

with a trait BayesC $\pi$ and BayesBL are expected to perform better than BayesA and BayesGBLUP.

More investigation is required on how different prior densities i.e student's $t$ and Laplace and different hyperparameters or say hierarchical and non-hierarchical models influence estimates. In the present study, all hyperparameters were estimated with prior densities, but one may study the difference when they are prespecified. As there were no pedigree records, it was not possible to add a polygenic component in the present study, but it would be interesting to study models with both polygenic and marker effect hierarchical and non-hierarchical models.

\section{Conclusion}

From the current study, it could be concluded that Bayesians methods are very useful in estimating both genetic marker effects associated with QTLs and genomic breeding values and that they could be applied in practice for selecting young calves based on their genomic breeding values with reasonably high confidence just on having their genotypes without having any phenotypic records of their own.

\section{Acknowledgements}

The authors thank the NDDB management for allowing the publication of the work presented in this article. The financial support received from the Department of Animal Husbandry and Dairying, GoI, for the Gir project is greatly acknowledged.

\section{References}

Boison SA, Utsunomiya ATH, Santos DJA, Neves HHR, Carvalheiro R, Mészáros G, Utsunomiya YT, do Carmo AS, Verneque RS, Machado MA, Panetto JCC, Garcia JF, Sölkner J, da Silva MVGB (2017).
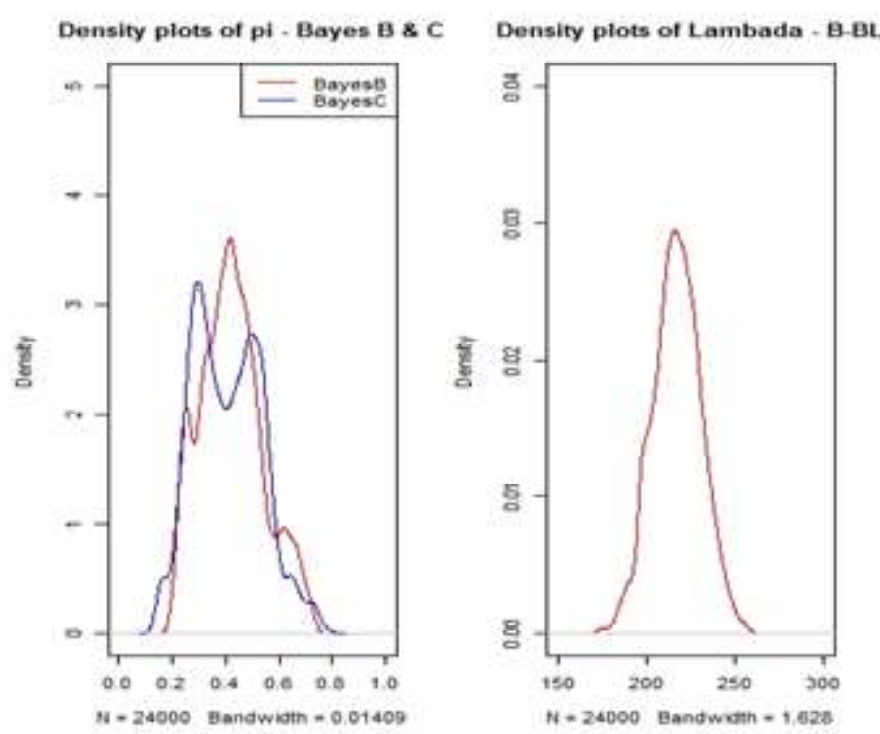

Accuracy of genomic predictions in Gyr (Bos indicus) dairy cattle. J Dairy Sci 100:5479-5490

Breed Survey (2013) Estimated livestock population breed wise based on breed wise survey. Ministry of Agriculture \& Farmers Welfare, Department of Animal Husbandry, Dairying and Fisheries, Government of India, New Delhi

Colombani C, Legarra A, Fritz S, Guillaume F, Croiseau P, Ducrocq V, Robert-Granié C (2013) Application of Bayesian least absolute shrinkage and selection operator (LASSO) and BayesCð methods for genomic selection in French Holstein and Montbéliarde breeds. J Dairy Sci 96:1-17

Daetwyler HD, Pong-ong R, Villanueva B, Woolliams JA (2010) The impact of genetic architecture on genome-wide evaluation methods. Genetics 185: 1021-1031

de los Campos G, Naya H, Gianola D, Crossa J, Legarra A, Manfredi E, Weigel K, Cotes JM (2009) Predicting quantitative traits with regression models for dense molecular markers and pedigree. Genetics 182:375-385

Habier DJ, Tetens F, Seefried R, Lichtner P, Thaller G (2010) The impact of genetic relationship information on genomic breeding values in German Holstein cattle. Genet Sel Evol 42:5

Hayes BJ, Chamberlain AJ, Maceachern S, Savin K, McPartlan H, MacLeod I, Sethuraman L, Goddard ME (2009) A genome map of divergent artificial selection between Bos Taurus dairy cattle and Bos taurus beef cattle. Anim Genet 40:176-184

Karkkainen HP, Sillanpaa MJ (2012) Back to basics for bayesian model building in genomic selection. Genetics 191: 969-987

Legarra, A, Robert-Granie C, Croiseau P, Guillaume F, Fritz S (2011) Improved Lasso for genomic selection. Genet Res 93:77-87

Meuwissen THE, Hayes BJ, Goddard ME (2001) Prediction of total genetic value using genome-wide dense marker maps. Genetics 157:18191829

Nayee N, Gajjar S, Sudhakar A, Saha S, Trivedi KR, Vataliya P (2020) Genomic Selection in Gir Cattle using female reference population. J Anim Sci 90: 1612-1616

Park T, Casella G (2008) The Bayesian Lasso. J Am Stat Assoc 103:681686.

Perez P, de los Campos MG (2014) Genome-wide regression and prediction with the BGLR statistical package. Genetics 198:483-495

Saha S, Nayee N, Shah H, Gajjar S, Kishore G, Gupta RO, Trivedi KR (2020) Effect of composition and size of the reference population 
in genotype imputation efficiency of INDUSCHIP in HF Crossbred cattle. Indian J Dairy Sci 73:250-255

Saha S, Nayee N, Sudhakar A, Gajjar S, Trivedi KR, Gupta RO, Kishore G. Evaluating Efficiency of Customized Medium Density (53K) INDUSCHIP Version 1 for Genotyping of Indicine Cattle Breeds (2020) Indian J Anim Sci 90:1476-1478

Schopke K, Swalve HH (2016) Review: Opportunities and challenges for small populations of dairy cattle in the era of genomics. Animal 10:1050-1060
Spiegelhalter DJ, Best NG, Carlin BP, Van Der Linde A (2002) Bayesian Measures of Model Complexity and Fit. J Royal Stat Society 64: 583-639

Verbyla KL, Hayes BJ, Bowman PJ, Goddard ME (2009) Accuracy of genomic selection using stochastic search variable selection in Australian Holstein Friesian dairy cattle. Genet Res 91:307-311 